\title{
MEAN VALUES FOR HOMOGENEOUS STIT TESSELLATIONS IN 3D
}

\author{
WERNER NAGEL ${ }^{1}$ AND VIOLA WEISS ${ }^{2}$ \\ ${ }^{1}$ Institut für Stochastik, Friedrich-Schiller-Universität Jena, D-07737 Jena, Germany; ${ }^{2}$ Fachhochschule Jena, FB \\ Grundlagenwissenschaften, Carl-Zeiss-Promenade 2, D-07745 Jena, Germany \\ e-mail: nagel@minet.uni-jena.de \\ (Accepted January 30, 2008)
}

\begin{abstract}
Recently (Nagel and Weiss, 2005), the class of homogeneous random tessellations that are stable under the operation of iteration (STIT) was introduced. In the present paper this model is reviewed and new results for the mean values of essential geometric features of STIT tessellations in two and three dimensions are provided and proved. For the isotropic model, these mean values are compared with those ones of the Poisson-Voronoi and of the Poisson plane tessellations, respectively.
\end{abstract}

Keywords: crack pattern, mean values, random tessellations, spatial statistics, stochastic geometry.

\section{INTRODUCTION}

The geometry of several structures that are studied in material science, biology and other sciences can appropriately be modeled by random tessellations. Examples are single-phase polyhedral microstructures, foams, systems of cracks (joints, fissures) in rock, craquelée of thin layers, systems of cells. There are physical theories explaining the genesis of such structures and resulting in geometric models. Sometimes such models are hardly treatable, as, e.g., in the case of the Gilbert model, see Noble (1967) or the model by Gray et al. (1976), cf. Stoyan et al. (1995). On the other hand, there are idealized mathematical models which are easier to deal with theoretically, but their goodness-of-fit in certain applications has to be checked thoroughly (cf. Nagel et al., 2007).

Two well-established mathematical models for random tessellations are the Poisson-Voronoi tessellation and the Poisson plane tessellation (in 3D) or Poisson line tessellation (in 2D), respectively, (cf. Stoyan et al., 1995). A further model is the socalled STIT tessellation, introduced in Nagel and Weiss (2005). Although it arose as a result of purely mathematical investigations, it will enrich the choice of models. The simulations in Figs. 2 and 3 suggest that STIT tessellations can be potential models for crack or fissure structures.

In the present paper, after a brief description of the STIT model and a review of some key properties, new results for mean values of important parameters are presented. This is done for the homogeneous (i.e., spatially stationary) but not necessarily isotropic case. These mean values are compared with the corresponding ones for Poisson plane (or line respectively) tessellations and for the Poisson-Voronoi tessellations. Since homogeneous Poisson-Voronoi tessellations are always isotropic this comparison is restricted to the isotropic case. The proofs for the mean value formulas are given in the Appendix.

In this paper, a tessellation is assumed to be a partition of the Euclidean space into bounded and convex polytopes - the so-called cells - with the additional condition of local finiteness, i.e., any bounded subset of the space intersects a finite number of cells. Alternatively, a tessellation is given by the set of all boundary points of the cells, i.e., the boundaries between the cells (which is a topologically closed set).

\section{STIT TESSELLATIONS}

The name STIT is an abbreviation for "stable with respect to iteration", and this will be explained below in this Section. We start with a short description of STIT tessellations that was already given in earlier papers (Nagel and Weiss, 2005; Nagel et al., 2007).

\section{DESCRIPTION OF THE MODEL}

Let $\mathbb{R}^{d}$ denote the $d$-dimensional Euclidean space. Here we consider the cases $d=2$ and $d=3$. A hyperplane is a $(d-1)$-dimensional plane in $\mathbb{R}^{d}$, i.e., a plane in $\mathbb{R}^{3}$ or a line in $\mathbb{R}^{2}$, respectively. A hyperplane $h$ can be described by the signed distance $p$ of $h$ from the origin and by its unit normal vector $u$ in the upper half-space, i.e., a vector $u$ in the upper unit half sphere $\mathscr{S}_{+}^{d-1}$ in $\mathbb{R}^{d}$. We define that $p>0$ if the intersection of $h$ and the orthogonal line through the origin, $h^{\vdash}$, is in the upper half-space and $p<0$ otherwise. A random hyperplane has a random direction and a random distance to the origin. 
Let $\Lambda$ be the measure on the set of hyperplanes that is given by

$$
\Lambda(\mathrm{d}(p, u))=\mathrm{d} p \mathscr{R}(\mathrm{d} u),
$$

where $\mathrm{d} p$ is the element of the Lebesgue (length) measure on the real axis, and $\mathscr{R}$ is a probability distribution on the space of directions. If $\mathscr{R}$ is the uniform distribution on $\mathscr{S}_{+}^{d-1}$ (and thus the measure $\Lambda$ is invariant under all rotations of hyperplanes around the origin) then it is called isotropic. Notice that for the following construction it is not necessary that $\mathscr{R}$ is isotropic. But in order to generate a tessellation with bounded polytopes as cells it is assumed that not all random hyperplanes are orthogonal to one single plane (in $\mathbb{R}^{3}$ ) or to one single line (in $\mathbb{R}^{2}$ ), respectively. For a set $C \subset \mathbb{R}^{d}$ denote by $[C]$ the set of all hyperplanes $h$ that hit $C$, i.e., $C \cap h \neq \emptyset$. Thus, if $C$ is bounded, then $\Lambda([C])$ is finite and

$$
\Lambda_{C}(\cdot)=\frac{1}{\Lambda([C])} \Lambda(\cdot \cap[C]),
$$

defines a probability measure on $[C]$, i.e., the distribution of a random hyperplane that hits $C$.

Now let $W \subset \mathbb{R}^{d}$ be a $d$-dimensional compact and convex domain in which the STIT tessellation will be generated. Further assume that $\left(\tau_{j}, \gamma_{j}\right), j=1,2, \ldots$ is a sequence of independent and identically distributed (i.i.d.) pairs, $\tau_{j}, \gamma_{j}$ independent, $\tau_{j}$ exponentially distributed with parameter $\Lambda([W])$, and $\gamma_{j}$ a random hyperplane with distribution $\Lambda_{W}$. For a (random) hyperplane $\gamma$ denote by $\gamma^{+1}$ and $\gamma^{-1}$ the two halfspaces generated by $\gamma$. For $a>0$ the construction can now be described by the following algorithm.

\footnotetext{
$\operatorname{ALGORITHM}(a, W, \Lambda)$

0. $j=0, T=\{(0, W)\}, R=\emptyset$

1. UNTIL $T=\emptyset$ FOR $\left(\tau, W^{\prime}\right) \in T$ DO
}

(i) $j=j+1$

(ii) IF $\tau+\tau_{j} \leq a$ THEN

$$
\begin{array}{ll}
\text { (a) } & \text { IF } \gamma_{j} \in\left[W^{\prime}\right] \text { THEN } \\
& T=\left(T \backslash\left\{\left(\tau, W^{\prime}\right)\right\}\right) \cup\left\{\left(\tau+\tau_{j}, W^{\prime} \cap\right.\right. \\
& \left.\left.\gamma_{j}^{+1}\right),\left(\tau+\tau_{j}, W^{\prime} \cap \gamma_{j}^{-1}\right)\right\} \\
\text { (b) } & \operatorname{ELSE} T=\left(T \backslash\left\{\left(\tau, W^{\prime}\right)\right\}\right) \cup\left\{\left(\tau+\tau_{j}, W^{\prime}\right)\right\}
\end{array}
$$

(iii)ELSE $T=T \backslash\left\{\left(\tau, W^{\prime}\right)\right\}, R=R \cup\left\{W^{\prime}\right\}$

\section{END}

The OUTPUT of the algorithm is $R(a, W)=R$ which is a set of random convex polytopes in $W$ if $W$ itself is a convex polygon. This can be considered as a tessellation within $W$. We denote

$$
Y(a, W)=c l\left[\left(\bigcup_{W^{\prime} \in R(a, W)} \partial W^{\prime}\right) \backslash \partial W\right],
$$

where $\partial W^{\prime}$ denotes the boundary of the set $W^{\prime}$ and $c l$ the topological closure of a set. Thus $Y(a, W)$ is the union of all the chords inside $W$ which are generated by the $\gamma_{j}$ during the construction.

Roughly, this construction can be understood as follows. The set $W$ is subsequently divided at random times. After the first division at time $\tau_{1}$ by $\gamma_{1}$ the two 'cells' $W \cap \gamma^{+1}$ and $W \cap \gamma^{-1}$ arise. These two cells are treated separately and independently. Each cell has a life time until it is divided by a random hyperplane. The condition 'IF $\gamma_{j} \in\left[W^{\prime}\right]$ THEN' in the algorithm defines a rejection method (i.e., not all the $\gamma_{j}$ do divide a cell) which yields that the life time of a cell $W^{\prime}$ is exponentially distributed with the parameter $\Lambda\left(\left[W^{\prime}\right]\right)$, and hence in the average small cells live longer than larger ones. At the fixed time $a$ this procedure stops and the state of the construction defines $Y(a, W)$.

Fig. 1 illustrates the result of the construction of a non-isotropic tessellation with a small number of horizontal and vertical edges. In Figs. 2 and 3 simulations of isotropic STIT tessellations are shown.

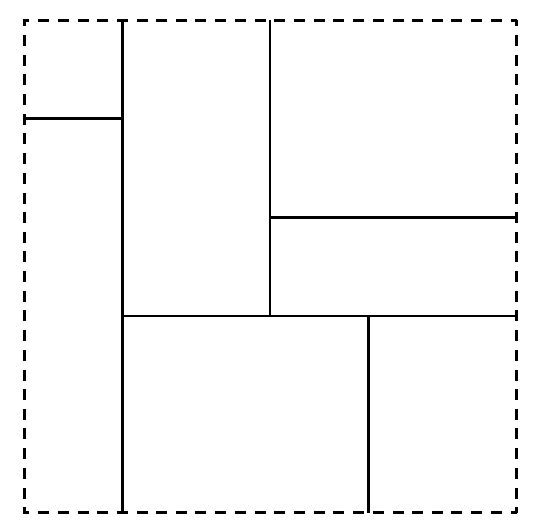

Fig. 1. Illustration of the construction with horizontal and vertical segments only.

\section{SOME KEY PROPERTIES}

Here we summarize important properties of the constructed tessellation. The proofs were already given in earlier papers (Nagel and Weiss, 2003; 2005). If $Y$ is a random tessellation of $\mathbb{R}^{d}$ then it is meant that $Y$ is the random closed set (RACS) of all cell boundaries. By $C(Y)$ we denote the set of all cells of $Y$. This is a random and space-filling ensemble of convex polytopes.

(i) Existence: There exists a homogeneous (i.e., spatially stationary) tessellation $Y$ of the whole $\mathbb{R}^{d}$ such that

$$
Y(a, W) \stackrel{D}{=} Y \cap W,
$$




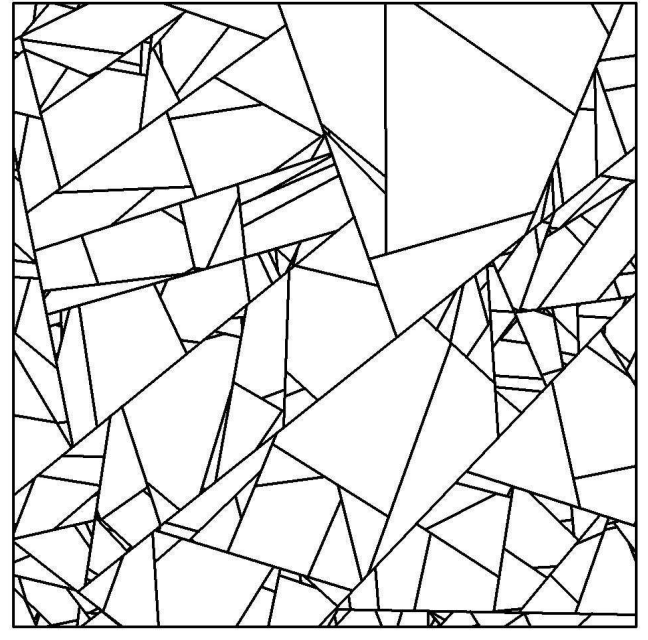

Fig. 2. Simulation of a 2D STIT tessellation (kindly provided by Joachim Ohser).

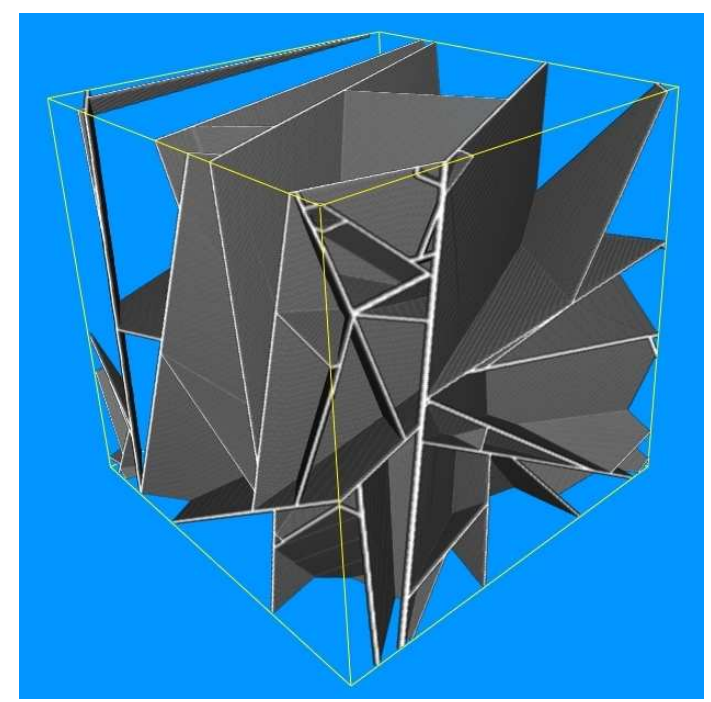

Fig. 3. Simulation of a 3D STIT tessellation (kindly provided by Joachim Ohser, Claudia Lautensack, and Tatyana Sych).

where $\stackrel{D}{=}$ stands for "has the same distribution as." The tessellation $Y$ does not depend on $W$, and one can show that this formula holds for all compact and convex $d$ dimensional sets $W \subset \mathbb{R}^{d}$. One possibility to construct also $Y$ is described in Mecke et al. (2007).

The directional distribution of the faces in $Y$ coincides with the distribution $\mathscr{R}$ that was introduced in Eq. 1. The intensities or densities of other parameters of the random tessellation depend on the value of $a$ in the construction above. For $d=2$ let $L_{A}$ be the mean total edge length per unit area and, respectively, for $d=3$ denote by $S_{V}$ the mean total face area per unit volume. Their values are

$$
\begin{array}{ll}
L_{A}=a & \text { if the construction is done in } \mathbb{R}^{2}, \\
S_{V}=a & \text { if the construction is done in } \mathbb{R}^{3} .
\end{array}
$$

(ii) STIT: This tessellation is stochastically stable with respect to the iteration of tessellations - STIT for short. For tessellations, the operation of iteration (also referred to as nesting) is defined as follows. Let $Y_{1}, Y_{2}, \ldots$ be a sequence of i.i.d. homogeneous random tessellations and denote $\mathscr{Y}=\left\{Y_{1}, Y_{2}, \ldots\right\}$. Further assume that $Y_{0}$ is a homogeneous random tessellation which is independent of $\mathscr{Y}$. For this definition it is useful to consider the set $C(Y)$ of the cells (which are convex polygons) of $Y$. Assume that these cells are numbered and that $C\left(Y_{0}\right)=\left\{p_{1}, p_{2}, \ldots\right\}$. The iteration of the tessellation $Y_{0}$ and the sequence $\mathscr{Y}$ is defined as

$$
I\left(Y_{0}, \mathscr{Y}\right)=Y_{0} \cup \bigcup_{k \geq 1}\left(p_{k} \cap Y_{k}\right) .
$$

This definition means that a cell $p_{k}$ of the so called "frame" tessellation $Y_{0}$ is - independently of all other cells - subdivided by the cells $p_{k i}, i=1,2, \ldots$ of the tessellation $Y_{k}$ which intersect the interior of $p_{k}$.

For a real number $r>0$ the tessellation $r Y$ is generated by transforming all points $(x, y) \in$ $Y$ into $(r x, r y)$. Accordingly, $r Y$ means that this transformation is applied to all tessellations of the sequence $\mathscr{Y}$.

Let $Y_{0}$ be a homogeneous random tessellation and $\mathscr{Y}_{1}, \mathscr{Y}_{2}, \ldots$ a sequence of sequences of tessellations such that all the occurring tessellations (including $\left.Y_{0}\right)$ are i.i.d. Then the sequence $I_{2}\left(Y_{0}\right), I_{3}\left(Y_{0}\right), \ldots$ of rescaled iterations is defined as (Nagel and Weiss, 2003; 2005).

$$
\begin{aligned}
I_{2}\left(Y_{0}\right) & =I\left(2 Y_{0}, 2 \mathscr{Y}_{1}\right) \\
I_{3}\left(Y_{0}\right) & =I\left(I\left(3 Y_{0}, 3 \mathscr{Y}_{1}\right), 3 \mathscr{Y}_{2}\right) \\
& =I\left(\frac{3}{2} I_{2}\left(Y_{0}\right), 3 \mathscr{Y}_{2}\right) \\
I_{m}\left(Y_{0}\right) & =I\left(\frac{m}{m-1} I_{m-1}\left(Y_{0}\right), m \mathscr{Y}_{m-1}\right), \\
& m=3,4, \ldots
\end{aligned}
$$

Here, $m$ is the rescaling factor which is chosen such that the results of tessellations do not degenerate for $m \rightarrow \infty$. We use the abbreviation $I_{m}\left(Y_{0}\right)$ since it is assumed that all the other tessellations in the sequences $\mathscr{Y}_{1}, \mathscr{Y}_{2}, \ldots$ are independent and have the same distribution as $Y_{0}$. The tessellation $I_{m}\left(Y_{0}\right)$ is generated by an $m$-fold iteration where all the used tessellations have the same distribution as $m Y_{0}$. 
Definition 1 A homogeneous random tessellation $Y$ is said to be stable with respect to iteration (STIT) if

$$
Y \stackrel{D}{=} I_{m}(Y) \quad \text { for all } m=2,3, \ldots,
$$

i.e., if its distribution is not changed by repeated rescaled iteration with sequences of tessellations with the same distribution.

It was shown in Nagel and Weiss (2005) that the tessellation $Y$ introduced in Eq. 3 is STIT. It should be emphasized that the STIT property uniquely determines the distribution of a homogeneous random tessellation if the edge length (or surface area) intensity and the directional distribution $\mathscr{R}$ are fixed. Roughly, this means that the above given construction yields the only STIT tessellations for $\mathscr{R}$.

(iii) Sections are STIT: If a homogeneous STIT tessellation $Y$ in $\mathbb{R}^{d}$ is intersected by a $k$-dimensional plane $h$, with $1 \leq k \leq d-1$, then the section profile $Y \cap h$ is again a homogeneous tessellation on this plane. The operations of iteration and of intersection commute, i.e.,

$$
I_{2}\left(Y_{0} \cap h, \mathscr{Y}_{1} \cap h\right)=I_{2}\left(Y_{0}, \mathscr{Y}_{1}\right) \cap h
$$

where $\mathscr{Y}_{1} \cap h=\left\{Y_{1} \cap h, Y_{2} \cap h, \ldots\right\}$.

Hence it is evident that $Y \cap h$ is a STIT tessellation if $Y$ is STIT.

(iv) Poisson typical cell: For homogeneous random tessellations the notion of the distribution of the random typical cell is defined via Palm distributions, see Stoyan et al. (1995) or Schneider and Weil (2000). Intuitively, the typical cell can be understood as a randomly chosen cell out of a finite number of cells, e.g., out of the set of all cells which have their centroid in a large ball around the origin. In such a choice all these finitely many cells have an equal probability to be selected; i.e., no weighting with respect to their size.

Now, let $Y^{P}$ denote a homogeneous Poisson hyperplane tessellation in $\mathbb{R}^{d}$ with intensity $L_{A}$ (if $d=2$ ) or $S_{V}$ (if $d=3$ ) respectively and directional distribution $\mathscr{R}$. And $Y$ is assumed to be a homogeneous STIT tessellation with the same parameter and the same $\mathscr{R}$. If one considers the interior of the cells or, more intuitively, the single isolated cells neglecting additional nodes or edges on their boundaries ( $c f$. the following paragraph (v)), one can show (Nagel and Weiss, 2003) that the distributions of the interiors of the typical cell of $Y^{P}$ and of $Y$ are identical.

(v) Cells are not face-to-face: In Voronoi tessellations as well as in hyperplane tessellations and in several other tessellation models the cells are in a face-to-face position (in Schneider and Weil (2000), the German word "seitentreu" is used). This means that for any two cells $C_{1}, C_{2}$ the intersection $C_{1} \cap C_{2}$ is either empty or is an $r$-dimensional face, $0 \leq r \leq$ $d-1$, which is a face of the polytope $C_{1}$ and a face of $C_{2}$. An example of two cells that are not in a face-to-face position is shown in Fig. 4. One can derive it from the construction that was described by the algorithm above, and one can observe it in the simulation example in Figs. 2 and 3 that not all the cells of STIT tessellations are in a face-to-face position. Thus on the boundary of a cell can appear additional nodes and edges. Therefore, it is essential to define carefully the notions of nodes, edges and faces for such tessellations.

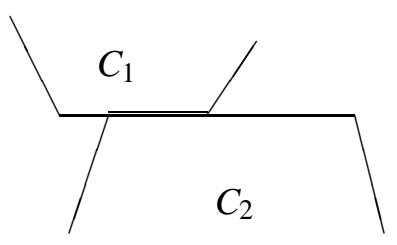

Fig. 4. Two cells $C_{1}$ and $C_{2}$ that are not in a face-toface position. Their intersection is the bold line, and this is not a face of $C_{1}$ or of $C_{2}$.

\section{MEAN VALUES FOR STIT TESSELLATIONS}

\section{THE PLANAR CASE $(d=2)$}

For the sake of completeness we recall here results from Nagel and Weiss (2004) for the planar case.

In a planar tessellation where the cells are compact convex polygons the set of the nodes is given as the set of all vertices of these polygons. All nodes of a STIT tessellation are so-called $T$-shaped nodes with exactly three emanating edges. In order to define the edges of the tessellation consider the network of all cell boundaries, i.e., the union of all segments which are faces of cells. Obviously, all the nodes are located on the network. An edge of the tessellation is a linear segment in this network between two nodes but with no node in between (i.e., no node is in the relative interior of this segment). Thus, an edge of the tessellation is always an intersection of two cells. In Fig. 4 the bold line illustrates one such edge of the tessellation. 

values.

We will use the following notation for mean

$$
\begin{aligned}
& L_{A} \quad-\quad \text { mean total edge length per unit area, } \\
& \text { edge length intensity, } \\
& U_{1} \quad-\text { mean length of the typical edge, } \\
& U_{2}, A_{2} \quad-\text { mean perimeter and mean area, resp., } \\
& \text { of the typical cell, } \\
& N_{0} \quad-\text { mean number of nodes per unit area, } \\
& N_{1} \quad-\text { mean number of edge midpoints per } \\
& \text { unit area, } \\
& N_{2} \quad-\quad \text { mean number of cell centroids per } \\
& \text { unit area, } \\
& N_{01}=N_{02}-\text { mean number of edges emanating } \\
& \text { from the typical node } \\
& =\text { mean number of cells which } \\
& \text { contain the typical node, } \\
& N_{20}=N_{21}-\text { mean number of nodes } \\
& =\text { mean number of edges on } \\
& \text { the boundary of the typical cell. }
\end{aligned}
$$

For two directions $u_{1}, u_{2} \in \mathscr{S}_{+}^{1}$ denote by $\left[u_{1}, u_{2}\right]=$ $\left|\sin \angle\left(u_{1}, u_{2}\right)\right|$ the area of the parallelogram which is spanned by these two unit vectors. For the directional distribution $\mathscr{R}$ as in Eq. 1 we denote

$$
\zeta=\iint\left[u_{1}, u_{2}\right] \mathscr{R}\left(\mathrm{d} u_{1}\right) \mathscr{R}\left(\mathrm{d} u_{2}\right) .
$$

In the isotropic case, i.e., when $\mathscr{R}$ is the uniform distribution, we have $\zeta=\frac{2}{\pi}$.

Mecke (1984) showed that all the mean values introduced above for planar homogeneous random tessellations can be expressed by three parameters, namely $N_{0}, N_{2}$ and $L_{A}$. For the particular case of homogeneous STIT tessellations with edge length intensity $L_{A}$ and directional distribution $\mathscr{R}$ it is

$$
N_{0}=L_{A}^{2} \zeta \quad \text { and } \quad N_{2}=\frac{1}{2} L_{A}^{2} \zeta .
$$

Thus the well-known mean value formulas yield for the remaining parameters

$$
\begin{aligned}
& U_{1}=\frac{2}{3 L_{A} \zeta}, \quad U_{2}=\frac{4}{L_{A} \zeta}, \quad A_{2}=\frac{2}{L_{A}^{2} \zeta}, \\
& N_{1}=\frac{3}{2} L_{A}^{2} \zeta, \\
& N_{01}=N_{02}=3, \quad N_{20}=N_{21}=6 .
\end{aligned}
$$

\section{THE SPATIAL CASE $(d=3)$}

In a tessellation in $\mathbb{R}^{3}$ where the cells are compact convex polytopes the set of the nodes is given as the set of all vertices of these polytopes. In order to define the faces and edges of the tessellation consider the 2network of all cell boundaries, i.e., the union of all 2faces of cells. Further, the 1-network is defined as the union of all 1-faces (edges) of the cells. Obviously, the set of nodes is a subset of the 1-network which is itself a subset of the 2-network. An edge of the tessellation is a linear segment in the 1-network between two nodes but with no intermediate nodes (i.e., no node is in the relative interior of this segment). A face of the tessellation is a two-dimensional convex polygon which is a subset of the 2-network and is bounded by edges of the 1-network and has no edges in its relative interior. Hence, a face of the tessellation is always the intersection of two cells.

We consider the following mean values.

$$
\begin{aligned}
& \text { Mean ... } \\
& L_{V} \quad \text { - total edge length per unit volume, } \\
& \text { edge length intensity, } \\
& S_{V} \quad-\text { total face area per unit volume, } \\
& \text { face area intensity, } \\
& U_{1} \quad-\quad \text { length of the typical edge, } \\
& U_{2}, A_{2} \quad \text { - perimeter and area, resp., } \\
& \text { of the typical face, } \\
& U_{3}, B_{3}, A_{3}, V_{3} \text { - total edge length, mean width, } \\
& \text { surface area and volume, resp., } \\
& \text { of the typical cell, } \\
& N_{0}, N_{1}, N_{2}, N_{3} \text { - number of nodes, of edge } \\
& \text { midpoints, of face centroids, } \\
& \text { of cell centroids, resp., } \\
& \text { per unit volume, } \\
& N_{01}, N_{02}, N_{03} \quad \text { - number of edges, of faces, of cells, } \\
& \text { resp., that meet in the typical node, } \\
& N_{12}=N_{13} \quad-\quad \text { number of faces, of cells, resp., } \\
& \text { that meet in the typical edge, } \\
& N_{20}=N_{21} \quad-\text { number of nodes, of edges, resp., } \\
& \text { on the boundary of the typical face, } \\
& N_{30}, N_{31}, N_{32} \text { - number of nodes, of edges, of } \\
& \text { faces, resp., } \\
& \text { on the boundary of the typical cell. }
\end{aligned}
$$

Also for the three-dimensional case, Mecke (1984) showed that all these mean values can be expressed by a system of 7 parameters, namely

$$
\begin{aligned}
& N_{0}, N_{3}, N=N_{1}+N_{2}, L_{V}, S_{V}, \\
& T=N_{03} N_{0}, Z=N_{2} U_{2}=N_{3} U_{3} .
\end{aligned}
$$

The parameter $T$ can be interpreted as the mean multiple number of nodes per unit volume, and the parameter $Z$ as the mean total multiple edge length per unit volume. The multiplicity of a node or of an edge is given by the number of the adjacent cells.

A plane $h$ in $\mathbb{R}^{3}$ can be parametrized by $p$ and $h^{\vdash}$, as it was already used in Eq. 1 . Denote by $\mathscr{S}_{+}^{2}$ the 
upper unit half-sphere in $\mathbb{R}^{3}$, and identify the lines $h^{\vdash}$ with the unit vector that is defined by the intersection $u=h^{\vdash} \cap \mathscr{S}_{+}^{2}$. Thus the directional distribution $\mathscr{R}$ can also be understood as a distribution on $\mathscr{S}_{+}^{2}$. We abbreviate

$$
\begin{aligned}
& \zeta_{2}=\iint\left[u_{1}, u_{2}\right] \mathscr{R}\left(\mathrm{d} u_{1}\right) \mathscr{R}\left(\mathrm{d} u_{2}\right), \\
& \zeta_{3}=\iiint\left[u_{1}, u_{2}, u_{3}\right] \mathscr{R}\left(\mathrm{d} u_{1}\right) \mathscr{R}\left(\mathrm{d} u_{2}\right) \mathscr{R}\left(\mathrm{d} u_{3}\right),
\end{aligned}
$$

where the integration is over $\mathscr{S}_{+}^{2}$ and $\left[u_{1}, u_{2}\right]$ denotes the area of the parallelogram spanned by $u_{1}$ and $u_{2}$ and $\left[u_{1}, u_{2}, u_{3}\right]$ denotes the volume of the parallelepiped spanned by $u_{1}, u_{2}$ and $u_{3}$. $\pi / 8$.

In the isotropic case we have $\zeta_{2}=\pi / 4$ and $\zeta_{3}=$

For a homogeneous STIT tessellation with face area intensity $S_{V}$ and directional distribution $\mathscr{R}$ the mean values are as follows.

$$
\begin{aligned}
N_{0} & =S_{V}^{3} \zeta_{3}, \\
N_{3} & =\frac{1}{6} S_{V}^{3} \zeta_{3}, \\
N & =\frac{19}{6} S_{V}^{3} \zeta_{3}, \\
L_{V} & =S_{V}^{2} \zeta_{2}, \\
T & =4 S_{V}^{3} \zeta_{3}, \\
Z & =3 S_{V}^{2} \zeta_{2} .
\end{aligned}
$$

The proof is given in the Appendix. Hence, the known formulas for mean values of homogeneous tessellations (see Mecke, 1984) yield for STIT tessellations

$$
\begin{aligned}
& N_{1}=2 S_{V}^{3} \zeta_{3}, \\
& U_{1}=\frac{1}{2 S_{V}} \frac{\zeta_{2}}{\zeta_{3}}, \\
& U_{2}=\frac{18}{7 S_{V}} \frac{\zeta_{2}}{\zeta_{3}}, \quad A_{2}=\frac{6}{7 S_{V}^{2}} \frac{1}{\zeta_{3}}, \\
& U_{3}=\frac{18}{S_{V}} \frac{\zeta_{2}}{\zeta_{3}}, \\
& A_{3}=\frac{12}{S_{V}^{2}} \frac{1}{\zeta_{3}}, \quad B_{3}=\frac{3}{2 S_{V}} \frac{\zeta_{2}}{\zeta_{3}}, \\
& N_{01}=4, \quad V_{3}=\frac{6}{S_{V}^{3}} \frac{1}{\zeta_{3}}, \\
& N_{12}=N_{13}=3, \\
& N_{20}=N_{21}=\frac{36}{7}, \\
& N_{30}=24, \quad N_{03}=4,
\end{aligned}
$$

Finally, denote by $\mathscr{R}_{1}$ the directional distribution of the edges in a STIT tessellation $Y$, more precisely, the length-weighted directional distribution of edges or directional distribution in a typical edge point, respectively. The corresponding directional distribution for a Poisson plane tessellation is denoted by $\mathscr{R}_{1}^{P}$. If $Y$ and $Y^{P}$ are homogeneous STIT and Poisson plane tessellations respectively which have identical directional distributions $\mathscr{R}$ of their faces then

$$
\mathscr{R}_{1}=\mathscr{R}_{1}^{P} .
$$

A proof is given in the Appendix.

\section{COMPARISON WITH OTHER TESSELLATIONS}

Now the mean values of different random homogeneous tessellations are compared: the STIT tessellations, Poisson-Voronoi tessellations and the Poisson line (in $\mathbb{R}^{2}$ ) or Poisson plane (in $\mathbb{R}^{3}$ ) tessellations, respectively. This will be restricted to the isotropic case. The notation for STIT will be as above, the values for the Poisson-Voronoi tessellation are indicated by an upper index $V$ and those ones for the Poisson line or plane tessellations by the upper index $P$, respectively.

\section{THE PLANAR CASE $(d=2)$}

In the planar and isotropic case we have the parameter $\zeta=2 / \pi$. In order to make the tessellations comparable we assume that $N_{2}=N_{2}^{P}=N_{2}^{V}$ which also implies that the mean areas of the typical cells of all three tessellations are the same. Then we obtain with the results in Mecke (1984) ( $c f$. the references to Miles and Santaló therein)

$$
\begin{aligned}
& 2 N_{0}^{P}=N_{0}=N_{0}^{V}, \\
& \frac{3}{2} N_{1}^{P}=N_{1}=N_{1}^{V}, \\
& A_{2}^{P}=A_{2}=A_{2}^{V}, \\
& U_{2}^{P}=U_{2}=\frac{\sqrt{\pi}}{2} U_{2}^{V}, \\
& \frac{3}{2} U_{1}^{P}=U_{1}=\frac{\sqrt{\pi}}{2} U_{1}^{V}, \\
& L_{A}^{P}=L_{A}=\frac{\sqrt{\pi}}{2} L_{A}^{V}, \\
& N_{01}^{P}=N_{02}^{P}=4, \quad N_{01}=N_{02}=N_{01}^{V}=N_{02}^{V}=3, \\
& N_{20}^{P}=N_{21}^{P}=4, N_{20}=N_{21}=N_{20}^{V}=N_{21}^{V}=6 .
\end{aligned}
$$

Since the distribution of the typical cell of a STIT tessellation is the same as that one of a Poisson 
line tessellation, also the respective mean values $U_{2}$ and $L_{A}$ coincide. On the other hand. The numbers $N_{0}, N_{1}, N_{i j}, i, j=0,1,2$, that express some features of the mutual arrangement of the cells are conform for STIT and Poisson-Voronoi.

\section{THE SPATIAL CASE $(d=3)$}

In the three-dimensional and isotropic case the parameter values are $\zeta_{2}=\pi / 4$ and $\zeta_{3}=\pi / 8$. In analogy to the planar case we assume that $N_{3}=N_{3}^{P}=$ $N_{3}^{V}$ which also implies that the mean areas of the typical cells of all three tessellations are the same. The formulas given in Mecke (1984) (partially with references to Miles and to Santaló) yield the following results. Per unit volume:

$$
\begin{aligned}
& 6 N_{0}^{P}=N_{0} \approx 0.887 \quad N_{0}^{V}, \\
& 4 N_{1}^{P}=N_{1} \approx 0.887 \quad N_{1}^{V}, \\
& \frac{7}{3} N_{2}^{P}=N_{2} \approx 0.901 \quad N_{2}^{V}, \\
& S_{V}^{P}=S_{V} \approx 0.853 \quad S_{V}^{V}, \\
& 2 L_{V}^{P}=L_{V} \approx 0.829 L_{V}^{V} .
\end{aligned}
$$

Typical cell:

$$
\begin{aligned}
V_{3}^{P} & =V_{3}=\quad V_{3}^{V}, \\
A_{3}^{P} & =A_{3} \approx 0.853 \quad A_{3}^{V}, \\
B_{3}^{P} & =B_{3} \approx 0.829 B_{3}^{V}, \\
\frac{3}{2} U_{3}^{P} & =U_{3} \approx 0.829 \quad U_{3}^{V} .
\end{aligned}
$$

Typical face:

$$
\begin{aligned}
& \frac{3}{7} \quad A_{2}^{P}=A_{2} \approx 0.946 A_{2}^{V}, \\
& \frac{9}{14} \quad U_{2}^{P}=U_{2} \approx 0.920 \quad U_{2}^{V} .
\end{aligned}
$$

Typical edge:

$$
0.5 U_{1}^{P}=U_{1} \approx 0.935 U_{1}^{V} .
$$

Adjacent to the typical node:

$$
\begin{array}{ll}
N_{01}^{P}=6, & N_{01}=N_{01}^{V}=4, \\
N_{02}^{P}=12, & N_{02}=N_{02}^{V}=6, \\
N_{03}^{P}=8, & N_{03}=N_{03}^{V}=4 .
\end{array}
$$

In the typical edge:

$$
N_{13}^{P}=4, \quad N_{13}=N_{13}^{V}=3 .
$$

For the typical face:

$$
N_{20}^{P}=4, \quad N_{20}=\frac{36}{7} \approx 5.143, \quad N_{20}^{V} \approx 5.228 .
$$

For the typical cell:

$$
\begin{aligned}
& N_{30}^{P}=8, \quad N_{30}=24, \quad N_{30}^{V} \approx 27.071, \\
& N_{31}^{P}=12, \quad N_{31}=36, \quad N_{31}^{V} \approx 40.606, \\
& N_{32}^{P}=6, \quad N_{32}=14, \quad N_{32}^{V} \approx 15.535 .
\end{aligned}
$$

As pointed out in (iv) above, the distributions of the interiors of the typical cells of the STIT tessellation and of the Poisson plane tessellation coincide if their intensities $S_{V}=S_{V}^{P}$ and their directional distributions are the same. Hence it is obvious that also the other 'metrical' (or scale dependent) values $V_{3}, A_{3}, B_{3}, U_{3}$ coincide.

On the other hand, the cells of the STIT tessellation are not 'face-to-face'. Hence on the faces of the STIT cells are further edges and nodes. The mean values $N_{i j}$ with $i>j, i=2,3$, are some quantitative expression for that, and thus they provide some information about the mutual arrangement of the cells within a tessellation. The results clearly show that in this respect isotropic STIT and Poisson Voronoi tessellations are rather close together and that significant differences appear to the mean values for isotropic Poisson plane tessellations.

These results can be generalized also for some homogeneous but not necessarily isotropic tessellations which are generated by an affine transformation of an isotropic one.

\section{APPENDIX}

Now the proofs are given for the Eqs. 5-11 for homogeneous (but not necessarily isotropic) STIT tessellations. This will be based on the following facts.

i) A STIT tessellation has a Poisson typical cell, see (iv) in the section on key properties.

ii) If $Y$ is a STIT tessellation, then it is identically distributed as $I(2 Y, 2 \mathscr{Y})$. The calculation of mean values of $I(2 Y, 2 \mathscr{Y})$, where the effect of iteration is taken into account, provides equations for the mean values of $Y$.

iii) If $N_{0}, L_{V}, S_{V}$ are the mean values of $Y$, then the respective mean values of $2 Y$ are $N_{0} / 8, L_{V} / 4, S_{V} / 2$.

The Poisson plane tessellation with $S_{V}$ and directional distribution $\mathscr{R}$ has $N_{3}^{P}=N_{0}^{P}=S_{V}^{3} \zeta_{3} / 6$. Due to (i) it is $V_{3}=V_{3}^{P}$ and hence $N_{3}=N_{3}^{P}$, and thus Eq. 6.

In order to show Eq. 8, observe that the network of edges of $I(2 Y, 2 \mathscr{Y})$ consists of edges of the 
frame tessellation $2 Y$ and edges of $\left(2 p_{k} \cap 2 Y_{k}\right)$ nested into the frame cell $2 p_{k}, k=1,2, \ldots, c f$. Eq. 4, and, additionally, further edges which are generated by the intersection of the two-dimensional faces of $2 p_{k}$ with two-dimensional faces of $2 Y_{k}$. In order to calculate the length intensity of those edges that are newly generated by iteration consider independent and stationary processes $\Phi_{1}, \Phi_{2}$ of two-dimensional faces in $\mathbb{R}^{3}$ with distributions $P_{1}, P_{2}$, directional distributions $\mathscr{R}_{1}, \mathscr{R}_{2}$ and intensities $S_{V}^{(1)}, S_{V}^{(2)}$ (with $0<S_{V}^{(1)}, S_{V}^{(2)}<$ $\infty)$, respectively. Analogously to Theorem 1.1. and Lemma 3.2 which were shown for fibre processes by Mecke (1981), one can assert also for processes of faces in $\mathbb{R}^{3}$ that

$$
\begin{gathered}
\iiint I_{B}(y) \mu_{\varphi_{1} \cap \varphi_{2}}(\mathrm{~d} y) P_{1}\left(\mathrm{~d} \varphi_{1}\right) P_{2}\left(\mathrm{~d} \varphi_{2}\right) \\
=S_{V}^{(2)} \iiint I_{B}(y)\left|\sin \left(u T_{y} \varphi_{1}-v\right)\right| \times \\
\quad \times \mathscr{R}_{2}(\mathrm{~d} v) \mu_{\varphi_{1}}(\mathrm{~d} y) P_{1}\left(\mathrm{~d} \varphi_{1}\right) \\
=S_{V}^{(1)} S_{V}^{(2)} \iint|\sin (u-v)| \mathscr{R}_{1}(\mathrm{~d} u) \mathscr{R}_{2}(\mathrm{~d} v),
\end{gathered}
$$

where $T_{y} \varphi=\varphi-y, u \varphi$ denotes the normal direction to $\varphi$ in the origin $\mathbf{o}$ (given that $\mathbf{o} \in \varphi$ ), $\mu_{\varphi}$ the area measure jointed by $\varphi, \mu_{\varphi_{1} \cap \varphi_{2}}$ the length measure corresponding to $\varphi_{1} \cap \varphi_{2}$ and $B$ a Borel set of volume 1 in $\mathbb{R}^{3}$. Any face of the frame tessellation $2 Y$ has two sides where independent tessellations of the sequence $2 \mathscr{Y}$ abut against. Hence the length intensity of the newly generated edges is twice the value of Eq. 12 with $\mathscr{R}_{1}=\mathscr{R}_{2}=\mathscr{R}$ and $S_{V}^{(1)}=S_{V}^{(2)}=\frac{S_{V}}{2}$. This yields

$$
L_{V}=\frac{L_{V}}{4}+\frac{L_{V}}{4}+2 \cdot\left(\frac{S_{V}}{2}\right)^{2} \cdot \zeta_{2}
$$

and this implies Eq. 8.

This equation also shows that the edge length intensity $2 \cdot\left(S_{V} / 2\right)^{2} \cdot \zeta_{2}$ of those edges which are additionally generated in iteration by the intersection of pairs of two-dimensional faces is $L_{V} / 2$. In any of these edges exactly three cells of $I(2 Y, 2 \mathscr{Y})$ are meeting. Further, since $Z=N_{2} U_{2}$ for $Y$, for $2 Y$ the value of the mean total multiple edge length per unit volume is $\frac{N_{2}}{8} \cdot 2 U_{2}=\frac{Z}{4}$. Thus, due to stability under iteration,

$$
Z=\frac{Z}{4}+\frac{Z}{4}+3 \cdot \frac{L_{V}}{2}
$$

which implies Eq. 10.

Proof of Eq. 11: Let $Y$ and $Y^{P}$ be homogeneous STIT and Poisson plane tessellations respectively which have identical directional distributions $\mathscr{R}$ of their faces. The direction of an edge in $\mathbb{R}^{3}$ is described by the unit vector (in the upper half space) that is parallel to the edge and thus by the corresponding point in $\mathscr{S}_{+}^{2}$. The probability measure $\mathscr{R}_{1}$ is defined on $\mathscr{S}_{+}^{2}$. Let be $A \subset \mathscr{S}_{+}^{2}$. Then $L_{V} \mathscr{R}_{1}(A)$ is the mean total length of all those edges of $Y$ which directions belong to $A$. For $Y^{P}$ the analogous value is $L_{V}^{P} \mathscr{R}_{1}^{P}(A)$. Let $\mathbf{1}\{\cdot\}$ denote the indicator function that is 1 if the condition in brackets \{\} is fulfilled and 0 otherwise. For the Poisson plane tessellation with face area intensity $S_{V}$ it can be calculated that

$$
\begin{aligned}
& L_{V}^{P} \mathscr{R}_{1}^{P}(A) \\
= & \frac{\left(S_{V}^{P}\right)^{2}}{2} \iint[u, v] \cdot \mathbf{1}\left\{\operatorname{span}(u, v)^{\perp} \in A\right\} \mathscr{R}(\mathrm{d} u) \mathscr{R}(\mathrm{d} v),
\end{aligned}
$$

where $\operatorname{span}(u, v)^{\perp}$ is the normal vector of the plane which is spanned by $u$ and $v$. It describes the direction of the line which appears as the intersection of two hyperplanes with normal vectors $u$ and $v$, respectively.

Now consider the edges of the tessellation $I(2 Y, 2 \mathscr{Y})$. Those are (a) the edges of the frame $2 Y$ and (b) the edges of $2 p_{k} \cap 2 Y_{k}$ (i.e., the cut-outs of $2 Y_{k}$ in $2 p_{k}$ ), $k=1,2, \ldots$, and, additionally, (c) those edges that are generated by iteration when a face of $2 Y$ intersects a face of $2 Y_{k}$. There are always two cells adjacent to any face of the frame tessellation, and into each of these cells independent tessellations are nested in. With (iii) this yields three items that correspond to (a), (b), (c), namely

$$
\begin{aligned}
& L_{V} \mathscr{R}_{1}(A) \quad \begin{array}{c}
=\frac{L_{V}}{4} \mathscr{R}_{1}(A)+\frac{L_{V}}{4} \mathscr{R}_{1}(A) \\
+2 \cdot\left(\frac{S_{V}}{2}\right)^{2} \times \\
\times \iint[u, v] \cdot \mathbf{1}\left\{\operatorname{span}(u, v)^{\perp} \in A\right\} \mathscr{R}(\mathrm{d} u) \mathscr{R}(\mathrm{d} v) .
\end{array}
\end{aligned}
$$

Since $S_{V}=S_{V}^{P}$ is assumed we obtain

$$
L_{V} \mathscr{R}_{1}(A)=\frac{L_{V}}{2} \mathscr{R}_{1}(A)+L_{V}^{P} \mathscr{R}_{1}^{P}(A) .
$$

and thus with $L_{V}=2 L_{V}^{P}$ follows $\mathscr{R}_{1}=\mathscr{R}_{1}^{P}$.

Now consider the nodes of $I(2 Y, 2 \mathscr{Y})$. There are the nodes of the frame tessellation $2 Y$ and the nodes of the cut-outs $\left(2 p_{k} \cap 2 Y_{k}\right)$ which appear when $2 Y_{k}$ is nested into the cell $2 p_{k}, k=1,2, \ldots$, of the frame, and additionally, 'new' nodes that are generated by iteration. These 'new' nodes appear on the edges of the frame $2 Y$ and on the faces of $2 Y$. A complete description is given by the following three cases.

(a) New nodes on the edges of the frame $2 Y$ : They are generated by an intersection of these edges with a 
face of a nested $2 Y_{k}$. Since there are exactly three cells adjacent to each edge of $2 Y$ the intersection formulas for homogeneous tessellations, (11) and (iii) yield the intensity (i.e., mean number per unit volume) of new nodes of type (a) as

$$
3 \cdot\left(\frac{S_{V}}{2}\right)^{3} \cdot \zeta_{3} .
$$

(b) New nodes on the faces of $2 Y$ which appear as the intersection of such a face with an edge of a nested $2 Y_{k}$. Since each face of the frame $2 Y$ has two adjacent cells, the intensity of nodes of type (b) is

$$
2 \cdot\left(\frac{S_{V}}{2}\right)^{3} \cdot \zeta_{3}
$$

(c) Nodes on the faces of $2 Y$ can also appear in the intersection $\left(2 p_{j} \cap 2 Y_{j}\right) \cap\left(2 p_{k} \cap 2 Y_{k}\right)$ if $p_{k}$ and $p_{j}$ are adjacent cells of the frame tessellation which have a common two-dimensional face. On this face the traces of faces of $2 Y_{k}$ and of $2 Y_{j}$ can intersect and thus generate a node. The intensity of such nodes is

$$
\left(\frac{S_{V}}{2}\right)^{3} \cdot \zeta_{3}
$$

Thus the total intensity of new nodes of type (a), (b) or (c) is $\frac{3}{4} S_{V}^{3} \zeta_{3}$. Summarizing the investigation for the nodes we obtain

$$
N_{0}=\frac{N_{0}}{8}+\frac{N_{0}}{8}+\frac{3}{4} S_{V}^{3} \zeta_{3},
$$

and thus Eq. 5. This also shows that the intensity of the "newly generated" nodes is $\frac{3}{4} N_{0}$.

Any of the nodes that are newly generated (as described above) in the iteration $I(2 Y, 2 \mathscr{Y})$ has exactly 4 adjacent cells and exactly 4 emanating edges. From $T=N_{0} N_{03}$ we conclude

$$
T=\frac{T}{8}+\frac{T}{8}+4 \cdot \frac{3}{4} N_{0}
$$

and thus Eq. 9. Since

$$
N_{0} N_{01}=\frac{N_{0}}{8} N_{01}+\frac{N_{0}}{8} N_{01}+4 \cdot \frac{3}{4} N_{0},
$$

we obtain $N_{01}=4$.
The mean value formulas in Mecke (1984), in particular $N_{0} N_{01}=N+N_{0}-N_{3}=2 N_{1}$ and $N_{0}-N_{1}+$ $N_{2}-N_{3}=0$ together with Eqs. 5 and 6 and $N_{01}=4$ yield

$$
N_{2}=\frac{1}{6} N_{0}+2 N_{0}-N_{0}=\frac{7}{6} N_{0},
$$

and hence Eq. 7.

\section{REFERENCES}

Gray NH, Anderson JD, Devine JD, Kwasnik JM (1976). Topological properties of random crack networks. Math Geol 8:617-26.

Mecke J (1981). Formulas for stationary planar fibre processes III - Intersections with fibre systems. Math Operationsfor Stat 12: 201-10.

Mecke J (1984). Parametric representation of mean values for stationary random mosaics. Math Operationsfor Stat 15: 437-42.

Mecke J, Nagel W, Weiss V (2007). A global construction of homogeneous planar STIT tessellations. Jenaer Schriften Math u Inf, http://www.minet.unijena.de/preprints/nagel_06-2/.

Nagel W, Mecke J, Ohser J, Weiss V (2007). A tessellation model for crack patterns on surfaces. Proceedings of the 12th International Congress for Stereology, 2007 Aug 30 - September 7; Saint Etienne, France.

Nagel W, Weiss V (2003). Limits of sequences of stationary planar tessellations. Adv Appl Prob (SGSA) 35: 12338.

Nagel W, Weiss V (2004). Crack STIT tessellations existence and uniqueness of tessellations that are stable with respect to iteration. Izvestija Nacionalnoj Akademii Nauk Armenii, Matematika 39: 84-114. Also: J Contemp Math Analysis 39: 62-84.

Nagel W, Weiss V (2005). The crack tessellations characterization of the stationary random tessellations which are stable with respect to iteration. Adv Appl Prob (SGSA) 37: 859-83.

Noble B (1967). Applications of Undergraduate Mathematics in Engineering. Macmillan: New York.

Schneider R, Weil W (2000). Stochastische Geometrie. Stuttgart, Leipzig: BG Teubner.

Stoyan D, Kendall WS, Mecke J (1995). Stochastic Geometry and its Applications. 2nd edn. Chichester: John Wiley \& Sons. 